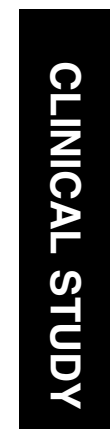

${ }^{1}$ Department of Ophthalmology, Bellvitge University Hospital, Barcelona, Spain

${ }^{2}$ Department of Ophthalmology, La Paz University Hospital, Madrid, Spain

${ }^{3}$ Department of Ophthalmology, Clínico San Carlos Hospital, Madrid, Spain

${ }^{4}$ Department of Ophthalmology, vall d'Hebron University Hospital, Universitat Autonoma de Barcelona, Barcelona, Spain

5INGO, Santiago de Compostela, Spain

${ }^{6}$ Department of Ophthalmology, Nuestra Señora de Valme University Hospital, Seville, Spain

${ }^{7}$ Department of Ophthalmology, Marques de Valdecilla University Hospital, Santander, Spain

${ }^{8}$ Department of Ophthalmology, La Fe University Hospital, Valencia, Spain

${ }^{9}$ Pfizer Inc, New York, NY, United States

Correspondence: L Arias, Department of Ophthalmology, Bellvitge University Hospital, C/Feixa Llarga, S/N, L'Hospitalet de Llobregat, Barcelona 08907, Spain

Tel: + 349326076 00; Fax: + 34914909749 . E-mail: luisarias@telefonica.net

Received: 24 April 2007 Accepted in revised form: 5 November 2007: Published online: 18 January 2008

\title{
Delay in treating age-related macular degeneration in Spain is associated with progressive vision loss
}

\begin{abstract}
Purpose To assess the impact on visual acuity of delays between diagnosis and treatment in patients with subfoveal neovascular agerelated macular degeneration (NV-AMD) and to evaluate NV-AMD patients' emotional status before therapy initiation.
\end{abstract}

Methods This retrospective, multicenter, epidemiological study included newly diagnosed NV-AMD patients registered in the Spanish national health system and referred to regional health centers for evaluation/ treatment by a retinal specialist from 09/2005 to $03 / 2006$. Records were reviewed and data abstracted at referring physicians' offices (diagnosis visit) and regional health centers (treatment visit). Treatment was at physicians' discretion. The Hospital Anxiety and Depression Scale was administered at the treatment visit (before therapy).

Results Median time from the diagnosis to treatment visit was 2.3 months $(\mathbf{9 5 \%}$ confidence interval: $\mathbf{0 . 2 - 1 0 . 8}$ months). Vision loss had progressed at the treatment visit with a doubling in the percentage of patients with a visual acuity of 20/400 or worse (from 12.4 to $24.7 \%$ ). The decrease in visual acuity from the diagnosis to the treatment visit was highly statistically significant $(P<\mathbf{0 . 0 0 0 1})$ as was the correlation between months to treatment and visual acuity change $(r=0.5234$, $P<0.0001)$. Time from the diagnosis to the treatment visit remained a significant predictor of progressive vision loss when visual acuity at diagnosis and change in lesion size between diagnosis and treatment were controlled $(P<\mathbf{0 . 0 0 0 1})$. Patients with more severe vision loss prior to treatment tended to report more depression.
L Arias ${ }^{1}$, F Armadá ${ }^{2}$, J Donate ${ }^{3}$, J García-Arumí ${ }^{4}$, J Giralt ${ }^{4}$, B Pazos ${ }^{5}$, A Piñero ${ }^{6}$, F Martínez ${ }^{7}$, JJ Mondéjar ${ }^{8}$, I Ortega ${ }^{2}$, G Zlateva ${ }^{9}$ and R Buggage ${ }^{9}$
Conclusions Delayed treatment of patients newly diagnosed with NV-AMD is associated with substantial visual acuity loss.

Eye (2009) 23, 326-333; doi:10.1038/sj.eye.6703053; published online 18 January 2008

Keywords: NV-AMD; visual acuity; treatment delay; Spain

\section{Introduction}

Age-related macular degeneration (AMD) is the main cause of severe and irreversible blindness in individuals $>50$ years of age worldwide and is the primary cause of legal blindness in Europe, Australia, and Japan. ${ }^{1}$ Of the two forms of the disease, atrophic and neovascular AMD (NV-AMD), the latter accounts for $10 \%$ of all AMD cases but is responsible for $90 \%$ of AMD-related severe vision loss. ${ }^{2,3} \mathrm{NV}$-AMD negatively affects both visual function and health-related quality of life ${ }^{4-9}$ with the reduction in quality of life being similar to such other chronic conditions as arthritis and chronic obstructive pulmonary disease. ${ }^{10}$

Early detection and treatment of NV-AMD not only can improve clinical outcomes ${ }^{11}$ but also can substantially reduce the personal, social, and economic burden of the disease. ${ }^{12,13}$ Delays can be experienced at any stage of treatment but may be most detrimental when they occur between the onset of symptoms and diagnosis and between diagnosis and initial treatment, the periods during which lesions may be most active and amenable to the benefits of therapy. In fact, a small study of 32 patients with NV-AMD treated in the Canadian health care system found that delays in assessing and 
treating new-onset disease by retinal specialists were associated with significant visual acuity losses. ${ }^{14}$

Although delays between the onset of symptoms and diagnosis in patient presentation may reflect a lack of awareness of NV-AMD and the availability of treatment options among the patients at risk for the disease, the delays experienced during the interval between diagnosis and treatment and their negative impacts may reflect the state of health care access, which was documented in a Canadian study. ${ }^{14}$ Patients in other systems with different pathways and gatekeepers to access may have longer or shorter delays to treatment and better or poorer patient outcomes. In Spain, for example, photodynamic therapy with verteporfin (PDT), which is indicated only for the treatment of predominantly classic and occult choroidal neovascular lesions, was, until recently, the single NV-AMD treatment approved for reimbursement by that country's national health authority. As a practical matter, access to PDT in Spain has been restricted both by its cost and by the fact that it can be administered only at a limited number of referral centers. Consequently, the number of patients who might benefit from PDT who actually receive treatment and the total number of treatments administered each year have been limited by local budgetary constraints. The impact of such constraints on patient outcomes has not been evaluated.

The primary objective of the present study was to assess visual acuity changes in patients with subfoveal NV-AMD treated in the Spanish health care system between diagnosis (diagnosis visit) and the initial visit with a retinal specialist for evaluation and treatment (treatment visit). (Note that the treatment visit was the point at which therapy could have been initiated; however, therapy might have been delayed in some cases.) In addition, each patient's emotional status at the treatment visit, before therapy initiation, was evaluated.

\section{Materials and methods}

This multicenter, retrospective, epidemiological study included patients diagnosed with subfoveal NV-AMD who were registered in the Spanish national health system and who were referred to any of 10 regional health centers for evaluation and treatment by a retinal specialist between September 2005 and March 2006. Ambulatory patients aged $\geqslant 50$ years of age of either gender with untreated subfoveal NV-AMD in one or both eyes were identified at the time of diagnosis upon referral to a regional health center for treatment. Patients were eligible for inclusion if they were capable of understanding and responding to study instruments and if they provided consent to participate. Exclusion criteria were diagnosis of choroidal neovascularization secondary to eye conditions other than NV-AMD; participation or planned participation in any other clinical trial during the study period; or clinical or psychological conditions the effects of which might interfere with the collection or interpretation of study findings. The study was approved by the Institutional Review Board of the San Carlos Clinical Hospital and by the Spanish Agency of Medicinal Products. The authors certify that all applicable institutional and governmental regulations concerning the ethical use of human volunteers were followed during this research.

Information concerning patient demographic and medical history, visual acuity, and lesion subtype, location, and size was abstracted from records at referring physicians' offices (diagnosis visit) and at regional health centers (treatment visit). Visual acuity in both eyes was measured using the Snellen chart at both visits. Given the observational nature of this study, decisions concerning treatment were made at the discretion of attending physicians, and no treatmentspecific or follow-up parameters were evaluated. At the treatment visit, prior to therapy initiation, the patient's emotional status was assessed using the Spanish version of the Hospital Anxiety and Depression Scale (HADS). The HADS contains 14 items, seven measuring anxiety and seven measuring depression. Individual item scores range from 0 to 3 ; total scores for both subscales are interpreted as normal (0-7), mild (8-10), moderate (11-14), and severe (15-21).

If both eyes were diagnosed with NV-AMD, the study eye was the first eye treated in the regional health center. For analyses, Snellen visual acuity scores were grouped into categories and assigned scores from 1 through 5 : normal, better than 20/40 (score $=1$ ); mildly limited, 20/40-20/80 (score =2); moderately limited, 20/80-20/200 (score $=3$ ); severely limited, 20/200-20/400 (score $=4$ ); and almost blind, 20/400 or worse (score $=5$ ). Change in visual acuity was measured as the visual acuity score at the treatment visit minus the visual acuity score at the diagnosis visit; thus, a positive result indicated progressive vision loss during the time period between the diagnosis and the treatment visits. The statistical significance of the mean change in visual acuity score between visits was evaluated using the Wilcoxon signed rank sum test for paired data. Spearman correlation was performed to assess the association between change in visual acuity and time to treatment. A multivariate linear regression model was developed to evaluate the association between time to treatment and change in visual acuity score while controlling for potential covariates; an ordinal logistic regression analysis (proportional odds model with cumulative logits) with visual acuity as an ordinal scale was also conducted. The objective was to obtain a sample of $100 \mathrm{NV}$-AMD patients 
representative of those treated for the condition at health centers from the public system in Spain; no formal power analysis was performed.

\section{Results}

\section{Demographic and clinical characteristics at diagnosis}

One hundred patients were enrolled. The mean patient age was $74.2 \pm 7.9$ years, half of patients were female, there was no family history of AMD in 84 of 93 (90.3\%) cases, 32 of 97 (33.0\%) had clear eyes or light iris color, and 45 of 99 $(45.4 \%)$ were smokers or former smokers. On average, patients had $2.2 \pm 1.5$ comorbidities; the most frequently reported were cataracts $(37 / 100(37.0 \%))$, vascular hypertensive disorders $(26 / 100(26.0 \%))$, and cardiac disease (25/100 (25.0\%)). At diagnosis, nearly half (46/95 $(48.4 \%))$ of study eyes had predominantly classic lesions, the majority $(59 / 89(66.3 \%))$ of lesions were subfoveal, and the average lesion size was $2.3 \pm 1.9$ disk areas.

\section{Treatment delay and vision loss}

The median time from the diagnosis visit to the treatment visit was 2.3 months (95\% confidence interval: $0.2-10.8$ months). Although half of the patients received treatment within 2.3 months, 25\% experienced delays of $>2.3-4.2$ months and an additional $25 \%$ had delays of >4.2-11.7 months.

At the time of diagnosis, 39 of $97(40.2 \%)$ study eyes had mildly or moderately limited visual acuity, 45 of 97 (46.4\%) eyes had severely limited visual acuity, and 12 of $97(12.4 \%)$ eyes were almost blind (Table 1$)$. At the treatment visit, vision loss had progressed, with a

Table 1 Visual acuity at diagnosis and treatment visits $(N=97)$

\begin{tabular}{lcc}
\hline Visual acuity level & $\begin{array}{c}\text { Visual acuity: } \\
\text { diagnosis visit } \\
\mathrm{n}(\%)\end{array}$ & $\begin{array}{c}\text { Visual acuity: } \\
\text { treatment visit } \\
\mathrm{n}(\%)\end{array}$ \\
\hline $\begin{array}{l}\text { 1. Normal }(>20 / 40) \\
\text { 2. Mildly limited } \\
(20 / 40-20 / 80)\end{array}$ & $14(1.0)$ & $1(1.0)$ \\
$\begin{array}{l}\text { 3. Moderately limited } \\
(20 / 80-20 / 200)\end{array}$ & $25(25.8)$ & $7(7.2)$ \\
$\begin{array}{l}\text { 4. Severely limited } \\
(20 / 200-20 / 400)\end{array}$ & $45(46.4)$ & $21(21.6)$ \\
$\begin{array}{l}\text { 5. Almost blind } \\
(<20 / 400)\end{array}$ & $12(12.4)$ & $44(45.4)$ \\
& & $24(24.7)$ \\
\hline $\begin{array}{l}\text { Visual acuity score } \\
\text { Change in visual }\end{array}$ & $3.5 \pm 0.9$ & $3.9 \pm 0.9$ \\
acuity score & & $0.3 \pm 0.7$ \\
$P$-value & & $<0.0001$ \\
\hline
\end{tabular}

$\mathrm{SD}$, standard deviation. doubling in the percentage of patients with a visual acuity of $20 / 400$ or worse or almost blind (24/97 $(24.7 \%))$. Between the diagnosis and treatment visits, visual acuity deteriorated in 27 of 95 (28.4\%) study eyes, improved in 5 of $95(5.3 \%)$ eyes, and remained stable in 63 of $95(66.3 \%)$ eyes. Vision loss occurred in 6 of 13 $(46.2 \%)$ eyes with mild vision loss at diagnosis, in 9 of 25 $(36.0 \%)$ eyes with moderate vision loss, and in 11 of 44 $(25.0 \%)$ eyes with severe vision loss. The decrease in visual acuity from the diagnosis to the treatment visit was highly statistically significant $(P<0.0001$; Table 1$)$ as was the correlation between months to treatment and visual acuity change ( $r=0.3534, P=0.0004$; Figure 1$)$.

Progression in vision loss by lesion subtype

Between the diagnosis and treatment visits, statistically significant progressive vision loss was observed in eyes with occult or minimally classic lesions that had higher mean visual acuity scores at the diagnosis visit $(P<0.03$ for both; Table 2), while a similar trend of vision loss $(P=0.0511)$ was also noted in eyes with predominantly classic lesions that had the worst mean visual acuity score at diagnosis. Visual acuity deteriorated in 10 of 45 $(22.2 \%)$ eyes with predominantly classic lesions, in 9 of $28(32.1 \%)$ eyes with occult lesions, and in 6 of $18(33.3 \%)$ eyes with minimally classic lesions. The percentage of eyes that were almost blind increased substantially between the diagnosis and treatment visits, at least doubling in eyes with occult and minimally classic lesions. The magnitude of vision loss was correlated with length of treatment delay in eyes with occult or minimally classic lesions but not in eyes with predominantly classic lesions.

\section{Progression in vision loss by lesion location}

Treatment delay negatively impacted visual acuity regardless of lesion location. Deterioration in visual acuity occurred in 18 of 57 (31.6\%) eyes with subfoveal lesions, in 3 of $24(12.5 \%)$ eyes with juxtafoveal lesions, and in 4 of $7(57.1 \%)$ eyes with extrafoveal lesions. Lesion location was stable in 88 of $89(98.9 \%)$ lesions classified; one lesion was classified as extrafoveal at the diagnosis visit but as juxtafoveal at the treatment visit.

Progression in vision loss by lesion size

The average lesion size increased between the diagnosis and treatment visits from $2.3 \pm 1.9$ to $2.6 \pm 1.7$ disc areas, respectively $(P=0.0412)$. Deterioration in visual acuity occurred in 13 of 38 (34.2\%) eyes with lesions $<1 \mathrm{~mm}$, in 5 of $21(23.8 \%)$ eyes with lesions $1-2 \mathrm{~mm}$, and in 7 of 30 (23.3\%) eyes with lesions $>2 \mathrm{~mm}$ in size. At diagnosis, lesion size and visual acuity score were not significantly correlated in patients with any lesion subtype (Table 3a). Progressive vision loss between the diagnosis and 


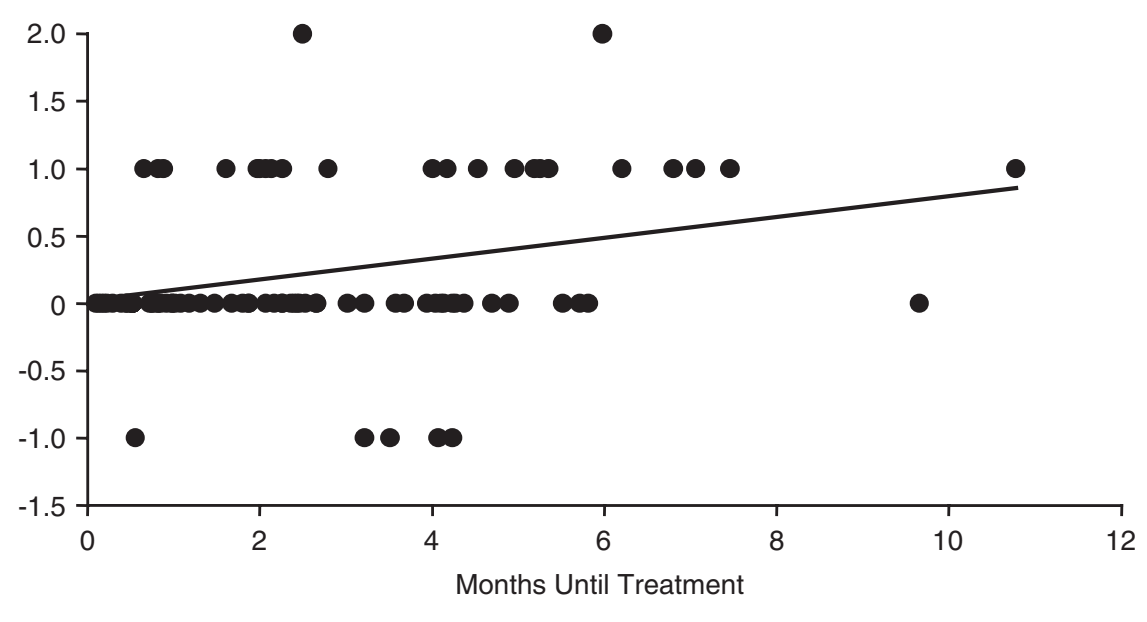

\begin{tabular}{lccc}
\hline & Change in Visual Acuity Score & \multicolumn{2}{c}{ Correlation } \\
\multicolumn{1}{l}{ Time to Treatment } & Mean $\pm \mathrm{SD}$ & $\mathrm{r}$ & $P$ Value \\
\hline$<1$ month $(\mathrm{N}=29)$ & $0.1 \pm 0.4$ & 0.2338 & 0.2222 \\
1 t o 2 months $(\mathrm{N}=12)$ & $0.2 \pm 0.4$ & 0.3735 & 0.2578 \\
2 to 3 months $(\mathrm{N}=18)$ & $0.4 \pm 0.6$ & -0.1874 & 0.4714 \\
$>3$ months $(\mathrm{N}=39)$ & $0.4 \pm 0.9$ & 0.6338 & 0.0001 \\
\hline \multicolumn{2}{c}{ Global correlation: $\mathrm{r}=0.3534 ; P=0.0004$} \\
\end{tabular}

Figure 1 Correlation between months to treatment and mean change in visual acuity score $(N=98)$.

Table 2 Visual acuity outcomes at treatment visit by lesion subtype $^{a}$

\begin{tabular}{|c|c|c|c|}
\hline & $\begin{array}{c}\text { Predominantly } \\
\text { classic } \\
\mathrm{N}=45\end{array}$ & $\begin{array}{c}\text { Occult } \\
\mathrm{N}=30^{\mathrm{b}}\end{array}$ & $\begin{array}{c}\text { Minimally } \\
\text { classic } \\
\mathrm{N}=18\end{array}$ \\
\hline \multicolumn{4}{|l|}{ Visual acuity score } \\
\hline Diagnosis visit & $3.8 \pm 0.8$ & $3.2 \pm 0.9$ & $3.6 \pm 1.1$ \\
\hline Treatment visit & $3.9 \pm 0.8$ & $3.5 \pm 1.1$ & $4.1 \pm 0.9$ \\
\hline Change in visual acuity score & $0.2 \pm 0.5$ & $0.3 \pm 0.7$ & $0.5 \pm 0.9$ \\
\hline$P$-value & 0.0511 & 0.0262 & 0.0242 \\
\hline \multicolumn{4}{|l|}{ Eyes almost blind, $n(\%)$} \\
\hline Diagnosis visit & $6(13.3)$ & $2(6.7)$ & $4(22.2)$ \\
\hline Treatment visit & $9(20.0)$ & $5(17.2)$ & $8(44.4)$ \\
\hline \multicolumn{4}{|c|}{$\begin{array}{l}\text { Correlation between change in visual acuity score and months to } \\
\text { treatment }\end{array}$} \\
\hline$r$ & 0.1546 & 0.4421 & 0.6628 \\
\hline$P$-value & 0.3105 & 0.0185 & 0.0027 \\
\hline
\end{tabular}

${ }^{a}$ Mean \pm standard deviation unless otherwise noted.

${ }^{\mathrm{b}}$ Data are missing for one patient at treatment visit.

treatment visits was significantly correlated with lesion size in eyes with predominantly classic and minimally classic lesions but not in eyes with occult lesions.
Regression model of treatment delay and progressive vision loss

When visual acuity at diagnosis and change in lesion size between diagnosis and treatment were controlled, time from diagnosis to treatment remained a statistically significant predictor of progressive vision loss $(P<0.0001$; Table $3 b)$. Visual acuity score at diagnosis was negatively associated with change in visual acuity score because eyes with severely limited vision had a smaller margin for deterioration while those with milder limitations had greater margins for change. Similar results were obtained with an ordinal logistic regression analysis.

\section{Emotional status at treatment visit}

At the treatment visit, a somewhat smaller percentage of patients reported being anxious than depressed, with 75 of $99(75.8 \%)$ scoring in the normal range on the anxiety HADS subscale and 65 of $99(65.7 \%)$ scoring in this range on the depression subscale. Patients with more severe vision loss at the treatment visit tended to report more depression (Figure 2). 
Table 3 Bivariate and multivariate analyses of visual acuity score predictors

a. Correlation between lesion size and visual acuity score by lesion subtype Visual acuity score: diagnosis visit

\section{Lesion subtype}

Predominantly classic, $N=45$

Occult, $N=29$

Minimally classic, $N=18$

Overall sample

Visul acl

b. Regression model explaining variability in change in visual acuity score $(\mathrm{N}=87)$

\begin{tabular}{lcrr}
\hline Variable & Parameter estimate & t-value & P-value \\
\hline Intercept & 0.813 & 3.06 & 0.0030 \\
Time to treatment (months) & 0.0361 & 4.32 & $<0.0001$ \\
Visual acuity score at diagnosis & -0.199 & -2.92 & 0.0046 \\
Change in lesion size & 0.091 & 2.21 & 0.0302 \\
Overall model: $R^{2}=0.391 ; \mathrm{F}(3,83)=17.772 ; P<0.0001$ & & \\
\hline
\end{tabular}

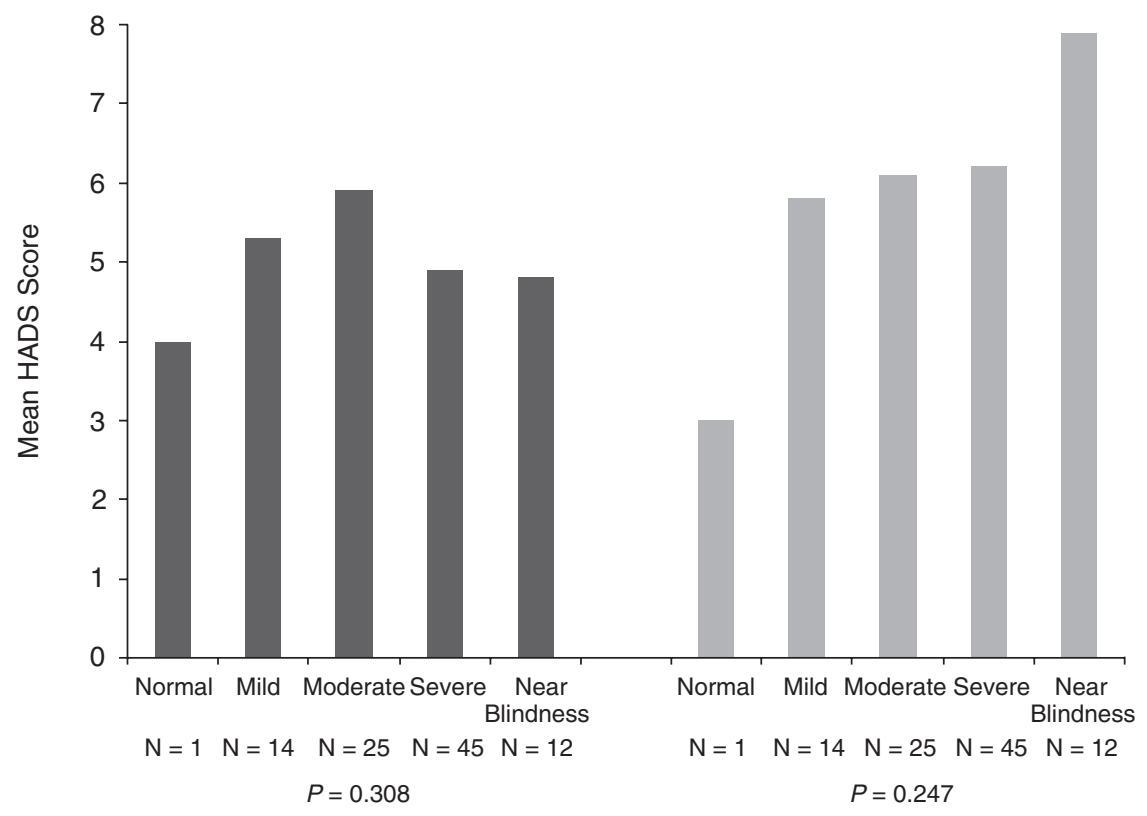

Figure 2 Hospital Anxiety and Depression Scale (HADS) scores by visual acuity at the treatment visit.

\section{Discussion}

Delayed treatment of patients newly diagnosed with NV-AMD is associated with substantial visual acuity loss. In the median of 2.3 months between diagnosis and treatment, vision loss occurred in 27 of 95 (28.4\%) study eyes, and the percentage of eyes that were almost blind doubled. The longer the treatment was delayed, the more the vision deteriorated, a relationship that remained when potential confounders were controlled. Progressive vision loss was also demonstrated when the sample was stratified by lesion morphology.

It is likely that our findings underestimate the magnitude of the relationship between treatment delay and vision loss because relatively small but potentially clinically meaningful changes in vision might not have 
been reflected by changes in our crude five-category outcome measure based on Snellen scores. The potential extent of the underestimation is suggested by results of a study conducted in a small number of patients treated in the Canadian health care system. ${ }^{14}$ Although a median of only 28 days elapsed between initial diagnosis and assessment/treatment, 14 of $32(43.8 \%)$ patients in the Canadian study had some degree of vision loss as measured by changes in the logarithm of the minimum angle of resolution (log MAR) compared to $28.4 \%$ of patients noted to have progressive vision loss over a median of 2.3 months in the present study. Change in $\log$ MAR is a more sensitive continuous measure of vision change that unfortunately was not routinely available in medical records in Spain.

Differences in outcome measures, sample sizes, or health care system features may explain discrepancies in the results of subanalyses conducted in the Canadian and current research. In the Canadian study, ${ }^{14}$ eyes with predominantly classic lesions were more likely than eyes with other lesion subtypes to lose a significant amount of vision $(P<0.002)$, and no relationship between lesion size and progressive vision loss was found. In contrast, we found a marginally statistically significant decline in visual acuity in those with predominantly classic lesions but highly significant vision loss in eyes with occult and minimally classic lesions. With regard to lesion size, we found that deterioration in visual acuity was significantly correlated with lesion size in eyes with predominantly classic and minimally classic lesions but not in eyes with occult lesions. Studies with larger number of eyes with NV-AMD using sensitive measures of vision change may be helpful in further clarifying the details of the relationship between treatment delay and vision loss and to relate such findings to characteristics of the health care systems studied.

Despite differences in details, our overall findings and those of Oliver-Fernandez et $a l^{14}$ demonstrate that rapid vision loss occurs over short periods of time in a substantial proportion of newly diagnosed, untreated eyes with NV-AMD. These results add further weight to those of double-masked clinical trials in which eyes randomized to placebo or usual care experienced rapid vision loss. ${ }^{15-17}$ For example, $\geqslant 3$ lines of vision were lost after 3 months of follow-up by $29 \%$ of placebo-treated patients in the Treatment of Age-Related Macular Degeneration with Photodynamic Therapy study. ${ }^{16}$ In the VEGF-Inhibition Study in Ocular Neovascularization (V.I.S.I.O.N.) trial, ${ }^{17} 20 \%$ of patients receiving usual care, which could include PDT, lost at least three lines of vision at month 3 . A subgroup analysis of data from the V.I.S.I.O.N. trial found that outcomes in patients with early disease treated with usual care experienced vision loss similar to those in the control group of the overall study population; however, patients with early disease treated with pegaptanib experienced significantly better vision outcomes, allowing the authors to conclude that early treatment may lead to greater gain and preservation of vision in patients with NV-AMD. ${ }^{11}$

Although rapid access to treatment appears to be key to preserving vision in patients with NV-AMD, patients in Spain face several barriers to preserving vision. First, the lack of awareness of NV-AMD among the public and the lack of screening for NV-AMD by non-retina specialists may lead to substantial delays between the onset of symptoms and diagnosis. Second, those with NV-AMD face a delay of 2 weeks to many months between diagnosis and treatment during which time they are at substantial risk of losing additional visual acuity. Finally, PDT, indicated for the treatment of predominantly classic and occult lesions, was the only NV-AMD therapy approved when the study was initiated, and PDT could be administered only at a limited number of public centers. Other effective treatments for NV-AMD, such as pegaptanib, ${ }^{17-19}$ recently have been approved for reimbursement in Spain, but budgetary constraints may limit their use in the public health system.

These issues confront a large number of Spanish citizens. Although an ongoing, population-based study of the incidence or prevalence of NV-AMD in Spain will provide more accurate data, an analysis based on data from the Rotterdam trials ${ }^{20,21}$ has estimated that there were 122136 prevalent cases in Spain in 2006 and that there will be 162759 such cases in 2025 (Pfizer Inc, data on file). On the basis of our sample of 100 patients, the epidemiologic profile of patients in Spain appears generally to be similar to that of patients worldwide: the average age is $>70$ years, ${ }^{22}$ the majority of patients have $>1$ comorbidity, ${ }^{23}$ and most lesions referred for PDT are predominantly classic. Given the advanced age and associated medical conditions of many of these patients, early treatment with the most effective and safest possible medication is imperative to help them maintain their visual health.

A secondary objective of the study was to evaluate the emotional well being of NV-AMD patients at the time of treatment. Nearly one-quarter of these patients scored above the normal range, indicating a depressed state on the HADS depression subscale, a percentage somewhat lower than the $32.5 \%$ of individuals with advanced AMD found, by Brody et al, ${ }^{4}$ to have depressive disorder. The trend towards increased depression with more severe vision loss parallels results of previous studies linking AMD-related vision loss with reduced patient quality of life, increases in need for assistance with activities of daily living, as well as emotional distress and depression..$^{4-9}$ In the current study, the strength of the 
relationship between vision loss and depression might have been underestimated since the HADS may not be sensitive to capturing depression in non-institutionalized subjects. In addition, a sample approximately three times larger than that included here (ie, about 300 patients) would have been required to detect smaller but potentially important associations between vision loss and anxiety or depression.

This study has both strengths and limitations. Primary strengths are its relatively large sample, as compared to the small Canadian study, ${ }_{1}^{14}$ and the inclusion of an assessment of each patient's emotional status. The primary limitation of the study was the use of a categorical measure of visual acuity that likely led to an underestimation of actual vision loss. In addition, the study was limited by the facts that no vision-related parameters were assessed, no follow-up beyond the initial treatment visit was conducted, and no visionrelated quality of life measures were evaluated.

In conclusion, patients newly diagnosed with NV-AMD in Spain face substantial barriers to preserving vision. Changes in the Spanish health care delivery system designed to raise public awareness of NV-AMD, to implement screening for the condition, and to facilitate referral for early treatment with the most effective therapies available are critical to preventing or minimizing vision loss in these individuals.

\section{Acknowledgements}

This study was funded by Pfizer Inc., New York. Editorial support, including contributing to the development of the manuscript and styling the paper for journal submission, was provided by Dr Jane Murphy of Zola Associates and was funded by Pfizer Inc.

\section{References}

1 Ambati J, Ambati BK, Yoo SH, Sakurai E, Lynn BC, Kuziel WA et al. Age-related macular degeneration: etiology, pathogenesis, and therapeutic strategies. Surv Ophthalmol 2003; 48: 257-293.

2 Ferris III FL. Senile macular degeneration: review of epidemiologic features. Am J Epidemiol 1983; 118: 132-151.

3 Ferris III FL, Fine SL, Hyman L. Age-related macular degeneration and blindness due to neovascular maculopathy. Arch Ophthalmol 1984; 102: 1640-1642.

4 Brody BL, Gamst AC, Williams RA, Smith AR, Lau PW, Dolnak D et al. Depression, visual acuity, comorbidity, and disability associated with age-related macular degeneration. Ophthalmology 2001; 108: 1893-1900.

5 DeCarlo DK, Scilley K, Wells J, Owsley C. Driving habits and health-related quality of life in patients with age-related maculopathy. Optom Vis Sci 2003; 80: 207-213.
6 Mangione CM, Gutierrez PR, Lowe G, Orav EJ, Seddon JM. Influence of age-related maculopathy on visual functioning and health-related quality of life. Am J Ophthalmol 1999; 128: 45-53.

7 Rovner BW, Casten RJ, Tasman WS. Effect of depression on vision function in age-related macular degeneration. Arch Ophthalmol 2002; 120: 1041-1044.

8 Scott IU, Feuer WJ, Jacko JA. Impact of visual function on computer task accuracy and reaction time in a cohort of patients with age-related macular degeneration. Am J Ophthalmol 2002; 133: 350-357.

9 Williams RA, Brody BL, Thomas RG, Kaplan RM, Brown SI. The psychosocial impact of macular degeneration. Arch Ophthalmol 1998; 116: 514-520.

10 Chia EM, Wang JJ, Rochtchina E, Smith W, Cumming RR, Mitchell P. Impact of bilateral visual impairment on healthrelated quality of life: the Blue Mountains Eye Study. Invest Ophthalmol Vis Sci 2004; 45: 71-76.

11 Gonzales CR, VEGF Inhibition Study in Ocular Neovascularization (V.I.S.I.O.N.) Clinical Trial Group Enhanced efficacy associated with early treatment of neovascular age-related macular degeneration with pegaptanib sodium: an exploratory analysis. Retina 2005; 25 : 815-827.

12 Bonastre J, Le Pen C, Soubrane G, Qtel G. The burden of age-related macular degeneration: results of a cohort study in two French referral centres. Pharmacoeconomics 2003; 21: 181-190.

13 Hopley C, Salkeld G, Wang JJ, Mitchell P. Cost utility of screening and treatment for early age related macular degeneration with zinc and antioxidants. $\mathrm{Br} J$ Ophthalmol 2004; 88: 450-454.

14 Oliver-Fernandez A, Bakal J, Segal S, Shah GK, Dugar A, Sharma S. Progression of visual loss and time between initial assessment and treatment of wet age-related macular degeneration. Can J Ophthalmol 2005; 40: 313-319.

15 Macular Photocoagulation Study Group. Laser photocoagulation of subfoveal neovascular lesions in agerelated macular degeneration. Results of a randomized clinical trial. Arch Ophthalmol 1991; 109: 1220-1231.

16 Treatment of Age-Related Macular Degeneration with Photodynamic Therapy (TAP) Study Group. Photodynamic therapy of subfoveal choroidal neovascularization in agerelated macular degeneration with verteporfin: one-year results of 2 randomized clinical trials-TAP report no. 1. Arch Ophthalmol 1999; 117: 1329-1345.

17 Gragoudas ES, Adamis AP, Cunningham Jr ET, Feinsod M, Guyer DR, VEGF Inhibition Study in Ocular Neovascularization Clinical Trial Group. Pegaptanib for neovascular age-related macular degeneration. $N$ Engl J Med 2004; 351: 2805-2816.

18 VEGF Inhibition Study in Ocular Neovascularization (V.I.S.I.O.N.) Clinical Trial Group, D'Amico DJ, Masonson HN, Patel M, Adamis AP, Patel M et al. Pegaptanib sodium for neovascular age-related macular degeneration: two-year safety results of the two prospective, multicenter, controlled clinical trials. Ophthalmology 2006; 113: 992-1001.

19 VEGF Inhibition Study in Ocular Neovascularization (V.I.S.I.O.N.) Clinical Trial Group, Chakravarthy U, Adamis AP, Cunningham Jr ET, Goldbaum M, Guyer DR et al. Year 2 efficacy results of 2 randomized controlled clinical trials of pegaptanib for neovascular age-related macular degeneration. Ophthalmology 2006; 113: 1508-1521. 
20 Klaver CC, Assink JJ, van Leeuwen R, Wolfs RC, Vingerling $\mathrm{JR}$, Stijnen $\mathrm{T}$ et al. Incidence and progression rates of age-related maculopathy: the Rotterdam Study. Invest Ophthalmol Vis Sci 2001; 42: 2237-2241.

21 Vingerling JR, Dielemans I, Hofman A, Grobbee DE, Hijmering M, Kramer CF et al. The prevalence of age-related maculopathy in the Rotterdam Study. Ophthalmology 1995; 102: 205-210.
22 American Academy of Ophthalmology. Preferred Practice Pattern: Age-Related Macular Degeneration. American Academy of Ophthalmology: San Francisco, 2006, p 4.

23 Shah S, Zlateva G, Zhou S, Javitt JC. Comparison of comorbid conditions between wet AMD patients and a control cohort in the Medicare Population. Invest Ophthalmol Vis Sci 2006; 47 (E-abstract 2209). 